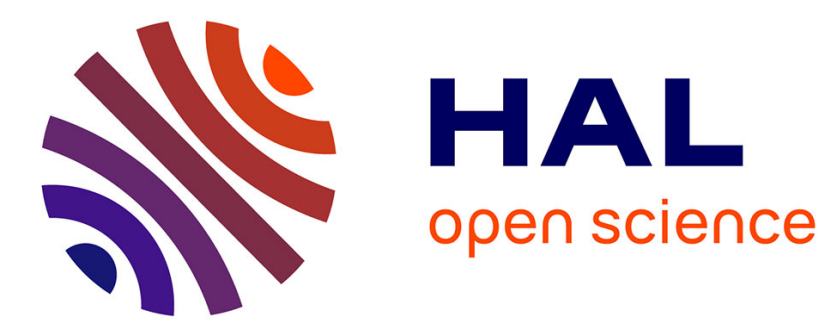

\title{
Photoacoustic angular resonance spectroscopy for characterizing thin films
}

\author{
H.-P. Shao, S.-Y. Zhang, Y.-S. Jing
}

\section{To cite this version:}

H.-P. Shao, S.-Y. Zhang, Y.-S. Jing. Photoacoustic angular resonance spectroscopy for characterizing thin films. Journal de Physique IV Proceedings, 1994, 04 (C7), pp.C7-615-C7-618. 10.1051/jp4:19947145 . jpa-00253203

\section{HAL Id: jpa-00253203 https://hal.science/jpa-00253203}

Submitted on 1 Jan 1994

HAL is a multi-disciplinary open access archive for the deposit and dissemination of scientific research documents, whether they are published or not. The documents may come from teaching and research institutions in France or abroad, or from public or private research centers.
L'archive ouverte pluridisciplinaire HAL, est destinée au dépôt et à la diffusion de documents scientifiques de niveau recherche, publiés ou non, émanant des établissements d'enseignement et de recherche français ou étrangers, des laboratoires publics ou privés. 


\title{
Photoacoustic angular resonance spectroscopy for characterizing thin films
}

\author{
H.-P. Shao, S.-Y. Zhang and Y.-S. Jing \\ Institute of Acoustics and Lab. of Modern Acoustics, Nanjing University, Nanjing 210008, China
}

\begin{abstract}
The photoacoustic angular resonance spectroscopy combining both the angular resonance and photoacoustic techniques is presented, which gives several advantages comparing with the traditional methods. The mechanism of the photoacoustic angular resonance spectroscopy for simultaneously determining the thickness and the refractive index of a film is discussed with extended contents. It is also demonstrated that the technique has special capabilities in quantitative non-destructive evaluation of thin films.
\end{abstract}

\section{INTRODUCTION}

With the development of the semiconductor technology and integrated optics, as well as the arising of various new materials, to determine the properties, such as the thicknesses and refractive indexes of thin films, becomes more and more important. A number of techniques have been used to determine the thicknesses and refractive indexes of films, there are ellipsometry[1], reflectance spectroscopy[2], waveguide technique[3], and resonance ATR technique[4], etc.. In this paper we extend a new developed photoacoustic angular resonance spectroscopy[5], combining the hemicylinder-film coupling structure with the photoacoustic absorption measurement technique, to obtain the thicknesses and refractive indexes of thin films simultaneously. Besides, concerning some thick films we present a straightforward photoacoustic angular resonance technique without the hemicylinder coupler to simplify the experimental arrangement and make the operation much easier. On the other hand, using the theory of electro-magnetic waves, we get the expressions of the stable guidewave modes and give the numerical calculations based on the given parameters in the experiment. The experimental results of this technique are consistent with the theoretical calculations, which shows the technique is either feasible or successful. At last, because the photoacoustic angular resonance spectroscopy put the merits of the waveguide technique, resonance ATR technique and the photoacoustic absorption technique together, we indicate that it is a new advantageous technique to characterize thin films.

\section{THEORY}

We proposed two methods to characterize thin and thick films respectively:

1.For thin films, the hemicylinder-film coupling photoacoustic absorption structure, shown in 
Fig. 1, is adopted. There are two cases to be discussed as follows

a. When $\theta_{0}$ is greater than the critical angle $\theta_{c}$ at the hemicylinder-air interface $\left(\theta_{c}=\arcsin \left(1 / n_{1}\right)\right)$, the incident light is totally reflected at the bottom of the hemicylinder, and the evanescent waves are excited in the air gap, which are exponentially decreasing in the direction perpendicular to the bottom of the hemicylinder. If the air gap is thin enough (typically, $t_{1}$ is less than or in the order of half a wavelength), the incident energy can be coupled into the film through the "optical tunneling" and the film behaves as a waveguide. The stable guidewave modes will be excited in the film as the incident angle $\theta_{0}$ satisfies the following equation[6]

$$
2 b_{2} t_{2}-2\left[\psi_{21}+\sin \left(2 \psi_{21}\right) \cos \left(2 \psi_{10}\right) \exp \left(-2 p_{1} t_{1}\right)\right]-2 \psi_{23}=2 m \pi
$$

where

$$
b_{2}=\sqrt{\left(k n_{2}\right)^{2}-\beta^{2}}, \quad p_{1}=\sqrt{\beta^{2}-\left(k n_{1}\right)^{2}}, \quad \beta=k n_{0} \sin \theta_{0},
$$

$k$ is the light wavenumber in air, $n_{i}$ and $t_{i}$ are the refractive index and the thickness of the $i$ th layer, $\psi_{i j}$ is half the reflection phase angle at the $i-j$ interface, $i, j=0,1,2,3$ denote the hemicylinder, air gap, film and substrate respectively, $m=0,1,2,3 \ldots$ is the integer denoting the modes of the guidewaves. If $n_{3}$ is less than $n_{2}$, the light beam will be totally reflected or partly penetrated (as a leaky waveguide) at the 2-3 interface, which depends on the incident angle at the 2-3 interface. If $n_{3}$ is greater than $n_{2}$, the film is a leaky waveguide. Then the phase difference between two neighboring light beams penetrating into the substrate is $2 \pi$. As a result, the photoacoustic signal from the film and the substrate increases to a maximum.

b. When $\theta_{0}$ is smaller than $\theta_{c}$, the incident light passes through the air gap directly, the

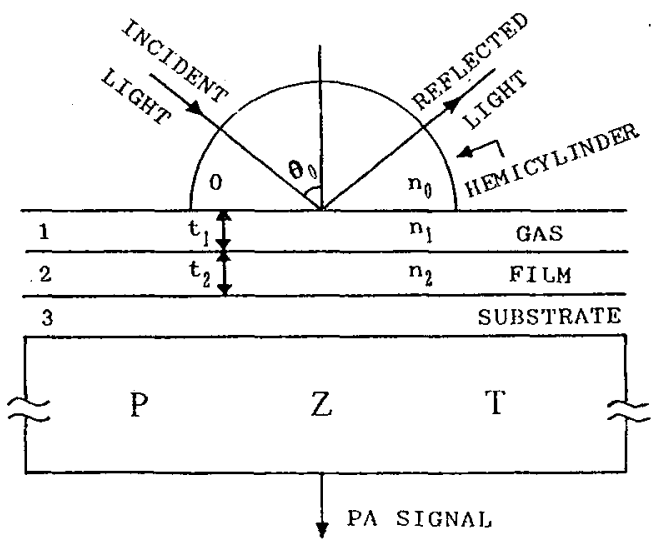

Fig. 1. Schematic diagram of the experimental arrangement. photoacoustic signal is proportional to the absorbed optical energy $V_{P A}$

$$
V_{P A}=1-\frac{r_{01}+r_{12} \exp \left(-2 i \delta_{1}\right)+r_{23} \exp \left(\left[-2 i\left(\delta_{1}+\delta_{2}\right)\right]+r_{01} r_{12} r_{23} \exp \left(-2 i \delta_{2}\right)\right.}{1+r_{01} r_{12} \exp \left(-2 i \delta_{1}\right)+r_{01} r_{23} \exp \left[-2 i\left(\delta_{1}+\delta_{2}\right)\right]+r_{12} r_{23} \exp \left(-2 i \delta_{2}\right)}
$$

where $r_{i j}$ is the reflectance at the $i-j$ interface, $\delta_{1}=\mathrm{kn}_{1} \mathrm{t}_{1} \cos \theta_{1}$ and $\delta_{2}=\mathrm{kn}_{2} \mathrm{t}_{2} \cos \theta_{2}$. If $\theta_{0}$ is the angle location of the peak in the spectrum, then

$$
\partial V_{P A} / \partial \theta_{0}=0
$$

There are three unknown parameters, i.e the thickness and refractive index of the film, as well as the width of the air gap, in the Eq.(1) and (3). If we obtain the photoacoustic angular absorption spectrum with three or more than three peaks, then the three parameters can be determined simultaneously. 
If the three or more than three peaks in the spectrum are located at the angles greater than the critical angle $\theta_{c}$, the order of the guidewaves is $m=0,1,2 \ldots$ from the right to left respectively, we can only use the Eq. (1) to obtain the result. If the number of the peaks with the angles greater than $\theta_{c}$ is less than three, but the total number of the peaks in the spectrum is three or more than three, using the Eq. (3) or combining the Eq. (1) with Eq. (3), the result can also be yielded.

If the films are very thin, there may exist only one or two peaks in the spectrum. We can estimate the width of the air gap by controlling the pressure of the hemicylinder against the film, using some additional restrictive conditions, we can obtain the values of the three parameters with the "N-M simplex method"[7].

2.For thick films, such as the thickness $t_{1}>1.5 \mu \mathrm{m}$ for $\mathrm{SiO}_{2}$ films on $\mathrm{Si}$ substrates if the incident light wavelength is about $500 \mathrm{~nm}$, we can take off the hemicylinder in Fig. 1 to make the operation much easier. The light beam illuminates the film directly, and the angle locations of the peaks in the photoacoustic angular absorption spectrum satisfy the equation

$$
2 t k \sqrt{n^{2}-\sin ^{2} \theta}-2 \psi=2 m \pi
$$

where $m$ is the integer number denoting the order of the guidewave modes in the leaky waveguide of the film, $t$ and $n$ are the thickness and the refractive index of the film respectively, $k$ is the wavelength of the incident light, and $\psi$ is half the reflection phase angle at the film and substrate interface. Since the value of $m$ is unknown, we should find at least 3 peaks in the spectrum if we want to yield the thickness and refractive index of the film simultaneously with the Eq. (4). If only two peaks appear in the spectrum, it can also be solved with the Eq. (3) assuming $n_{0}=1$.

\section{EXPERIMENT}

Either S-polarized (TE modes) or P-polarized (TM modes) incident light can be used in the experiment. It is worth indicating that we prefer to use S-polarized light than P-polarized light, since there are more and sharper peaks in the S-polarized spectrum than that with P-polarized light. Thus, the measurement precision is improved and the parameters of thinner films can also be determined when the S-polarized is utilized.

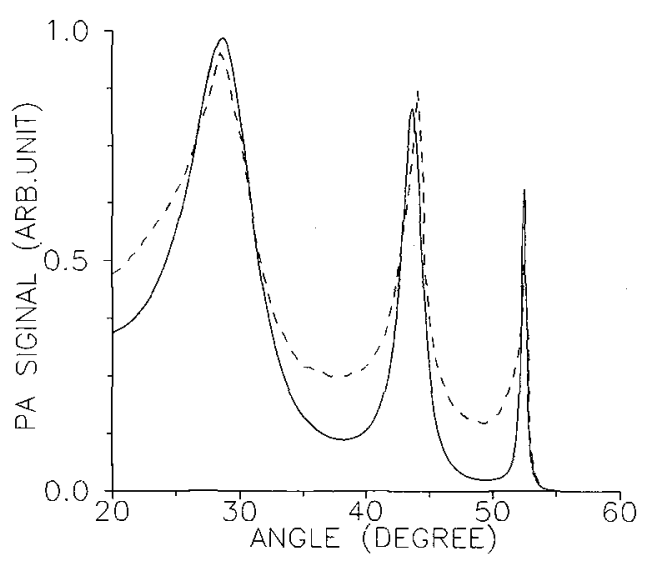

(a) $532 \mathrm{~nm}$, with the hemicylinder.

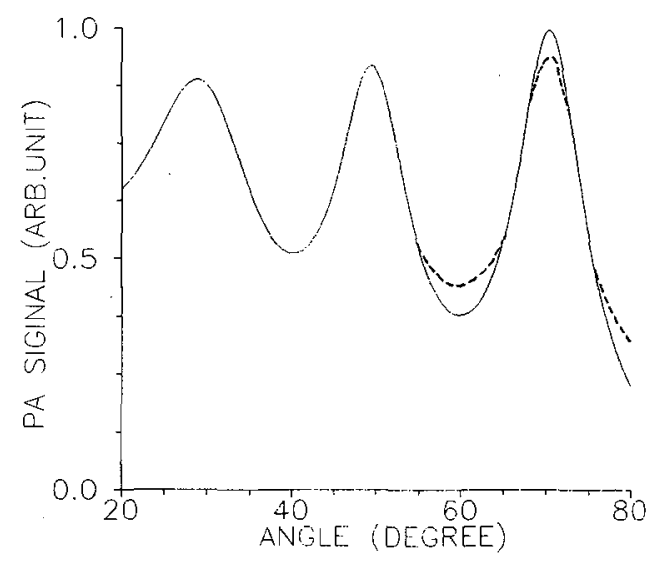

(b) $1860 \mathrm{~nm}$, without the hemicylinder

Fig. 2. The experimental and theoretical results for two different thickness film. 
We have measured several $\mathrm{SiO}_{2}$ films on $\mathrm{Si}$ substrates with various thickness, the S-polarized argon ion laser beam with the wavelength $488 \mathrm{~nm}$ and the hemicylinder with the refractive index 1.774 are used. The typical experiments for thin films with the hemicylinder coupler and for thick films without the hemicylinder, are shown in Fig.2. (a) and (b) respectively. In the figure, the dashed curves are obtained from the experiment and the solid curves are theoretically calculated by fitting to the experimental results. The measured results are $n_{2}=1.46, t_{2}=532 \mathrm{~nm}$ in (a), and $n=1.46, t=1860 \mathrm{~nm}$ in (b). The measured refractive indexes for several $\mathrm{SiO}_{2} / \mathrm{Si}$ films with different thickness by two different methods are in good agreement.

\section{CONCLUSION}

The photoacoustic angular resonance spectroscopy is developed on the waveguide and resonance ATR technique, but there are great differences between them. The information of the spectrum obtained by the incident angles less than the critical angle cannot be (or were not) utilized in both the waveguide and ATR techniques[3],[4]. However, the photoacoustic resonance absorption technique is specially suited to detect the weak signal, thus the measurement precision is improved, and the experimental arrangement is also simplified compared with the waveguide and ATR techniques, particularly when the thick films are measured without the hemicylinder coupler. Moreover, the extenuated photoacoustic angular resonance technique can characterize very thin films as well as thick films, such as the determination of the thicknesses and refractive indexes of films with high precision.

In order to enhance the detection capabilities of the photoacoustic angular resonance technique, it is much better to use the incident light with shorter wavelength and/or make the refractive index of the hemicylinder much greater than those of films. The further research works are in progress.

\section{REFERENCES}

[1] R. J. Archer, J. opt. soc. Am. 52 (1962) 970-977.

[2] F. Reizmen and W. Van. Gelder, Solid State Electron 10 (1967) 625-632.

[3] P. K. Tien, R. Ulrich and R. J. Martin, Appl. Phys. Lett. 14 (1969) 291-294.

[4] V. Bosachi and R. C. Oehrle, Appl. Opt. 21 (1982) 2167-2173.

[5] S. Y. Zhang, Y. C. Shen, Y. S. Jiang and R. Zhu, "Photoacoustic resonance absorption spectra of L-B films", Ultrasonics International '93, Vienna July 1993, (Edited by E.Benes) p. 178

[6] P. K. Tien and R. Ulrich, J. Opt. Soc. Am. 60 (1973) 1325-1337.

[7] A. Nealder and R. Mead, Computer J. 7, (1964) 308-311. 